\title{
Simulation analysis and optimization of fresh-slice multistage free-electron lasers
}

\author{
Zhaoheng Guo®, ${ }^{1,2, *}$ Marc W. Guetg $\odot,{ }^{1, *}$ Yuantao Ding, ${ }^{1}$ Agostino Marinelli, ${ }^{1,2}$ \\ Juhao Wu, ${ }^{1}$ Zhirong Huang, ${ }^{1,2}$ and Alberto A. Lutman $\oplus^{1, \dagger}$ \\ ${ }^{1}$ SLAC National Accelerator Laboratory, 2575 Sand Hill Road, Menlo Park, California 94025, USA \\ ${ }^{2}$ Stanford University, Stanford, California 94305, USA
}

(Received 4 November 2019; accepted 28 February 2020; published 17 March 2020)

\begin{abstract}
The generation of femtosecond soft x-ray free-electron laser (XFEL) pulses with an energy of hundreds of microjoules has been demonstrated at the Linac Coherent Light Source (LCLS) based on fresh-slice multistage amplifications. In this paper, we present a comprehensive simulation analysis and methods to improve fresh-slice multistage XFELs. A time-dependent transverse kick along the bunch is generated by two passive corrugated structures. The resulting oscillating orbit is controlled by downstream kickers, enabling different slices of the bunch to lase. The simulations reproduce the LCLS experimental results in terms of the pulse energy, bandwidth, and statistics of spectral spikes. The simulations reveal time-domain pulse profile properties, such as the pulse duration and structure, that were unavailable experimentally. We discuss possible issues connected with the accelerator setup and propose a simpler and more robust variation of the scheme to generate XFEL pulses shorter than $3 \mathrm{fs}$.
\end{abstract}

DOI: 10.1103/PhysRevAccelBeams.23.031304

\section{INTRODUCTION}

X-ray free-electron lasers (XFELs) [1-6], whose peak brightness is approximately 10 orders of magnitude higher than the third-generation light sources, are cutting-edge scientific instruments in chemistry, biology, atomic physics, material science, and other disciplines [7]. The development of advanced operational modes of XFELs has become an active field of investigation in order to satisfy requirements of various scientific applications.

The recent demonstration of fresh-slice lasing control [8] enhanced the power performance and delay control for multicolor operation over the basic split undulator concept [9], improved the power for self-seeded operation [10], and enabled production of high-power single spectral spike femtosecond pulses in the soft $x$ rays [11].

Different schemes have been proposed to selectively control the lasing process along the electron bunch while retaining the lasing capability in the temporal slices that do not participate in the lasing process [12-14]. After the

\footnotetext{
*zhaoheng@stanford.edu

aal@slac.stanford.edu

Present address: DESY, Notkestraße 85, D-22607 Hamburg, Germany.
}

Published by the American Physical Society under the terms of the Creative Commons Attribution 4.0 International license. Further distribution of this work must maintain attribution to the author(s) and the published article's title, journal citation, and DOI. initial demonstration [8], involving the transverse timedependent kick due to wakefields from a dechirper $[15,16]$, an energy chirp dispersion-based [17] and a timedependent matching scheme [18] have also been demonstrated. The highest-power soft x-ray FEL pulses are produced with dechirper-based multistage amplifications [8]. This method relies on the wakefield-induced timedependent dipole kick within dechirpers.

In this paper, we focus on the fresh-slice method based on a time-dependent kick imparted by passive wakefields of a corrugated metal jaw and reproduce the multistage amplification scheme in a simulation. In this scheme, an $\mathrm{x}$-ray pulse produced on the bunch tail is further amplified on fresh electrons in multiple stages. The results show the reliability of the simulation framework and give an estimate of the x-ray pulse duration and peak power that were unavailable in the original work, due to the lack of a diagnostic to measure the x-ray temporal profile (e.g., Ref. [19]). Electron-bunch-based measurements of the $\mathrm{X}$-ray pulse [20] fail when the same $\mathrm{X}$-ray pulse is amplified by different bunch slices in cascaded schemes or the slippage effects are significant. The pulse duration and peak power estimate is relevant information for experimental users that have already performed experiments or plan to use the multistage amplification scheme.

Our simulations expose issues related to the production of ultrashort pulses with the multistage scheme. Particularly harmful conditions could be an initial bunch tilt, the lack of full cancellation of the time-dependent focusing term, and the interplay between the cascaded passive structures from 
the time-dependent kick point of view. Finally, we show a simpler and more robust implementation of the scheme achieving a performance similar to the demonstrated results.

The paper is organized as follows. In Sec. II, we describe the machine layout relevant for the simulations and give information on how dechirpers have been modeled. In Sec. III, we simulate the multistage experiment reported in Ref. [11] using the Linac Coherent Light Source (LCLS) parameters. Effects of delays in chicanes and on-axis slice positions on XFELs are discussed. In Sec. IV, we propose a variation on the initial scheme that allows more robust performances with a simpler setup.

\section{MACHINE LAYOUT AND DECHIRPER MODEL}

The configuration of the LCLS is depicted in Fig. 1. A dechirper system is installed $120 \mathrm{~m}$ upstream of the undulator entrance to produce a strong short-range wake. An electron bunch traveling in the dechirper is subject to the transverse wakefields generated by the interaction with the metal plates. To kick the bunch transversely, it is more effective to use a single corrugated metal jaw, since the other jaw would cancel the kick produced in the first jaw. Therefore, in Fig. 2, we consider a single metal jaw for each dechirper module as it was used in the LCLS experiment [11]. Parameters of single-jaw dechirpers are listed in Table I. The vertical and the horizontal modules share the same design of the corrugated structure. The distance between the dechirper jaw and the electron bunch can be adjusted to control the transverse kick of the beam.

As a system of reference, we consider the bunch traveling along $z$, parallel to the machine's axis, at a velocity close to the speed of light $c$. We choose $z$ to describe the position of the electron in the beam line and $s=z-c t$ to describe the relative longitudinal position of the electron within the bunch. The tail of the bunch is located at $s=0$. We choose the center of the bunch before entering the dechirper system to be at $x=y=0$. We notice that the bunch is transversely kicked in the dechirper system and the bunch center will oscillate in the $x-y$ plane in the downstream beam line.

The horizontal corrugated jaw is located at $x=d_{x}$, as shown in Fig. 2. Similarly, the vertical one is located at $y=d_{y}$. We assume that the transverse size of the bunch $\sigma_{\perp}$ is small compared to the distances $d_{x}$ and $d_{y}$. For a leading particle at $(\tilde{x}, \tilde{y}, \tilde{s})$ and a test particle at $(x, y, s)$, the

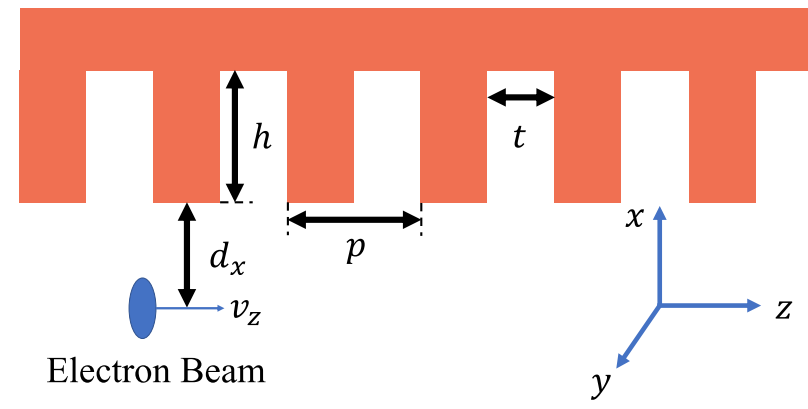

FIG. 2. The setup of the horizontal single-jaw dechirper. In the LCLS beam line, the $x-z$ plane is parallel to the ground. LCLS parameters of the dechirper setup are listed in Table I.

transverse wake function components in the horizontal dechirper are given by $[21,22]$

$$
\begin{aligned}
& w_{H, x}(x, y, \tilde{x}, \tilde{y}, \zeta)=w_{H, d}(\zeta)+(x-\tilde{x}) w_{H, q}(\zeta), \\
& w_{H, y}(x, y, \tilde{x}, \tilde{y}, \zeta)=-(y-\tilde{y}) w_{H, q}(\zeta),
\end{aligned}
$$

where $\zeta=\tilde{s}-s$ is the longitudinal distance between two particles. Here, $\zeta>0$ corresponds to the configuration where the leading particle is in front of the test particle in $z$. The dipole and the quadrupole wake functions of the single-jaw horizontal dechirper are given, respectively, by

$$
\begin{aligned}
& w_{H, d}(\zeta)=\frac{2}{d_{x}^{3}} s_{0 x}\left[1-\left(1+\sqrt{\frac{\zeta}{s_{0 x}}}\right) e^{-\sqrt{\zeta / s_{0 x}}}\right], \\
& w_{H, q}(\zeta)=\frac{3}{d_{x}^{4}} s_{0 x}\left[1-\left(1+\sqrt{\frac{\zeta}{s_{0 x}}}\right) e^{-\sqrt{\zeta / s_{0 x}}}\right],
\end{aligned}
$$

with $\zeta>0, s_{0 x}=8 d_{x}^{2} t /\left(9 \pi \alpha^{2} p^{2}\right)$, and $\alpha=1-0.465 \sqrt{t / p}-$ $0.07 t / p$. Parameters $d_{x}, p$, and $t$ are shown in Fig. 2, with LCLS parameters listed in Table I, and Eqs. (2) and (3) are valid for a bunch shorter than $100 \mu \mathrm{m}$ [22], which is satisfied under LCLS experimental conditions. The wake functions for the vertical dechirper are similar to the horizontal one:

$$
\begin{aligned}
& w_{V, x}(x, y, \tilde{x}, \tilde{y}, \zeta)=-(x-\tilde{x}) w_{V, q}(\zeta) \\
& w_{V, y}(x, y, \tilde{x}, \tilde{y}, \zeta)=w_{V, d}(\zeta)+(y-\tilde{y}) w_{V, q}(\zeta)
\end{aligned}
$$

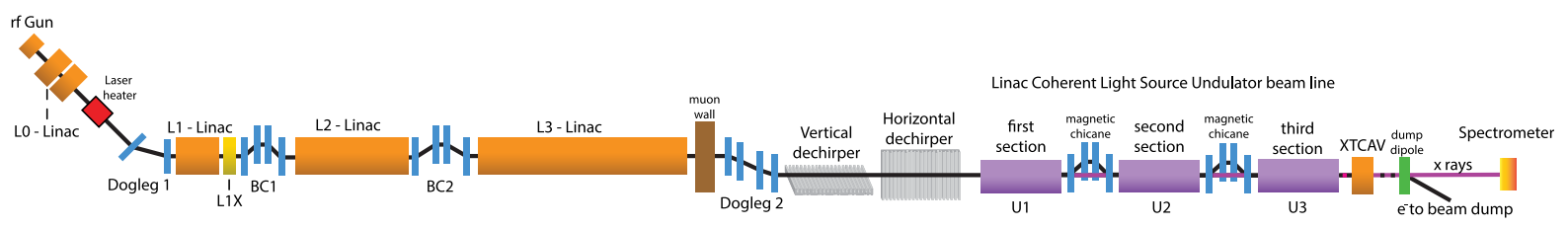

FIG. 1. The LCLS layout for fresh-slice three-stage XFEL pulse generation. Linac sections accelerate the electrons. The X-band transverse deflecting cavity (XTCAV) diagnoses the longitudinal phase space after the undulator beam line. L1X is the X-band linearizer cavity. $\mathrm{BC} 1$ and $\mathrm{BC} 2$ are the two bunch compressors. Dechirper and undulator sections are located downstream of the second dogleg. 
TABLE I. Main parameters used in simulations.

\begin{tabular}{lc}
\hline \hline Parameter & Value \\
\hline Dechirper & \\
Period $p$ & $500 \mu \mathrm{m}$ \\
Full depth $h$ & $500 \mu \mathrm{m}$ \\
Longitudinal gap $t$ & $250 \mu \mathrm{m}$ \\
Dechirper width $W$ & $12 \mathrm{~mm}$ \\
Dechirper length $L$ & $2 \mathrm{~m}$ \\
Electron bunch at undulator & \\
Projected normalized emittances $\epsilon_{n, x} / \epsilon_{n, y}$ & $1.14 / 0.65 \mu \mathrm{m}$ \\
$\quad$ without dechirper kick & \\
Beam energy $E$ & $3.9 \mathrm{GeV}$ \\
Bunch charge $Q$ & $140 \mathrm{pC}$ \\
Bunch length $L_{b}$ & $\sim 45 \mathrm{fs}$ \\
Peak current $I_{\text {peak }}$ & $3500 \mathrm{~A}$ \\
LCLS machine & \\
L1-S phase & $14.5^{\circ}$ \\
L1-X phase & $160^{\circ}$ \\
L2 phase & $35.8^{\circ}$ \\
BC1 $R_{56}$ & $-4.55 \mathrm{~cm}$ \\
BC2 $R_{56}$ & $-2.80 \mathrm{~cm}$ \\
Photon energy $E_{\text {XFEL }}$ & $670 \mathrm{eV}$ \\
Undulator period $\lambda_{u}$ & $3 \mathrm{~cm}$ \\
Twiss $\beta_{x} / \beta_{y}$ before undulator & $14.77 / 4.41 \mathrm{~m}$ \\
Twiss $\alpha_{x} / \alpha_{y}$ before undulator & $1.72 /-0.53$ \\
\hline \hline
\end{tabular}

where $w_{V, d}(\zeta)$ and $w_{V, q}(\zeta)$ are the same for the horizontal one but switching $d_{x}$ to $d_{y}$ in Eqs. (2) and (3).

As shown in Eqs. (1) and (4), the wakefield-induced kick in the transverse momentum of the test particle contains both the dipole term and the quadrupole term. The quadrupole term comes from the relative transverse offset $(x-\tilde{x}, y-\tilde{y})$ between the leading particle and the test particle, as previously studied in [23]. In the LCLS, the first dechirper is the vertical dechirper. The Coherent Synchrotron Radiation (CSR) that the bunch gets before the first dechirper affects $x$ direction and is usually smaller compared to the dipole kick produced in the dechirper system. To the first-order approximation in our dechirper study, we neglect the CSR effect and assume that the bunch enters the horizontal dechirper with all slices well centered, say, $\langle x(s)\rangle=\langle y(s)\rangle=0$ for all $s$. In this approximation, the whole quadrupole effect on the test particle can be described by an effective focal length $f_{V, q}(s)$. In this approximation, the vertical dechirper leads to a timedependent defocusing in $y$ and a time-dependent focusing effect in $x$.

By using both dechirper modules, we can alleviate the time-dependent quadrupole focusing effect along the beam as well as get a better FEL suppression with both horizontal and vertical dipole kicks. However, we should notice that, after being transversely kicked in the first dechirper, the bunch enters the second dechirper with slices off axis, leading to nonzero contributions from the quadrupole terms for the nonvanishing integrals over $\tilde{x}$ and $\tilde{y}$ in
Eqs. (1). This has an impact in the double-dechirper configuration. Detailed derivations of wakefield-induced transverse kick are given in the Appendix A.

\section{BENCHMARK SIMULATION WITH EXPERIMENT RESULTS}

In this section, we perform FEL simulations based on ELEGANT [24] and GENESIS version $4[25,26]$ to reproduce experimental data in Ref. [11]. We start from a particle distribution simulated with IMPACT [27] at the end of the injector and propagate it to the undulator entrance with ELEGANT. The horizontal and vertical dechirper elements have been included by implementing the wake functions in Eqs. (2) and (3). The code GENESIS version 4 has been used to simulate the FEL radiation in the undulator line with the input beam dumped from ELEGANT. Existing LCLS parameters listed in Table I have been used for the FEL simulations. When the dechirper jaws are fully retracted, the bunch at the undulator entrance is matched to Twiss parameters listed in Table I. The matching is achieved by optimizing the focusing strengths of the four matching quadrupole magnets. In the following, we refer to the nonkicked bunch with dechirper jaws retracted as the flat bunch. The FEL pulse energy of the matched flat bunch is $800 \mu \mathrm{J}$ at the end of $\mathrm{U} 1$ with the same undulator strengths used in the experiment [11], shown in Fig. 6 therein.

\section{A. Dechirper setup}

Because of uncertainties in the dechirper calibration in experiments, accurate values of $d_{x}$ and $d_{y}$ in Ref. [11] are not available. Therefore, we study the impact of the dechirper on the downstream bunch trajectory and transverse phase space distribution to replicate the experimental results.

By moving a dechirper jaw closer to the bunch, the stronger dipole kick in the bunch results in a better lasing suppression and benefits the generation of short XFEL pulses. The x-ray spectrum is expected also to present a narrower bandwidth by shortening the pulse in the time domain (effectively removing bandwidth broadening due to the electron bunch chirp), up to being a single spike when full longitudinal coherence is achieved. However, smaller $d_{x}$ and $d_{y}$ make time-dependent quadrupole effects also stronger and may also introduce higher-order effects. The FEL performance suffers from the mismatching effect produced by time-dependent quadrupole effects in dechirpers.

To discuss the time-dependent mismatching effect, we use the 4D betatron mismatching factor $\Phi(s)$. $\Phi(s)$ describes the mismatching between Twiss parameters of the slice at $s$ and the machine design [28]:

$$
\Phi(s)=\frac{1}{4} \operatorname{Trace}\left[\Sigma_{d}^{-1} \Sigma_{b}(s)\right],
$$

where the subscript $d$ refers to the designed lattice optics and $b$ to the bunch. The normalized bunch covariance matrix $\Sigma_{b}(s)$ of the slice $s$ is given by 


$$
\Sigma_{b}(s)=\left(\begin{array}{cccc}
\beta_{x}(s) & -\alpha_{x}(s) & 0 & 0 \\
-\alpha_{x}(s) & \gamma_{x}(s) & 0 & 0 \\
0 & 0 & \beta_{y}(s) & -\alpha_{y}(s) \\
0 & 0 & -\alpha_{y}(s) & \gamma_{y}(s)
\end{array}\right) .
$$

The matrix $\Sigma_{d}$ is defined in the same way but using Twiss parameters of the design optics. $\Phi(s)$ equals 1 for a particular slice at $s$ when the bunch slice and the machine are exactly matched. We consider $\Phi(s)$ at the entrance of the undulator beam line.

In Fig. 3, we show the averaged mismatching factor $\bar{\Phi}=\int d s I(s) \Phi(s) / \int d s I(s)$ with different dechirper system setups. $I(s)$ is the current profile, and the averaging integral is calculated for slices contributing to the FEL radiation in the undulator. Since the Twiss parameters of the whole beam are matched to the machine ones when the dechirper jaws are fully retracted, a deviation of $\bar{\Phi}$ from 1 measures how the lasing area of the bunch is mismatched to the undulator lattice.

We can see a good cancellation of the mismatching effect in the diagonal part of Fig. 3. As shown in Eq. (A3), the reciprocal time-dependent focal length of the dechirper is given by the convolution between the dechirper quadrupole wake function and the longitudinal density distribution of the bunch. With $d_{x} \simeq d_{y}$, the wake functions in two dechirpers are similar, and the focal lengths in two dechirpers are close to each other but with opposite sign. When $1 /\left|f_{V, q}(s)\right| \simeq 1 /\left|f_{H, q}(s)\right|$, the mismatching effect induced in the vertical dechirper can be compensated well by the downstream horizontal one.

Getting the best cancellation of the mismatching effect may not be enough when trying to produce the shortest

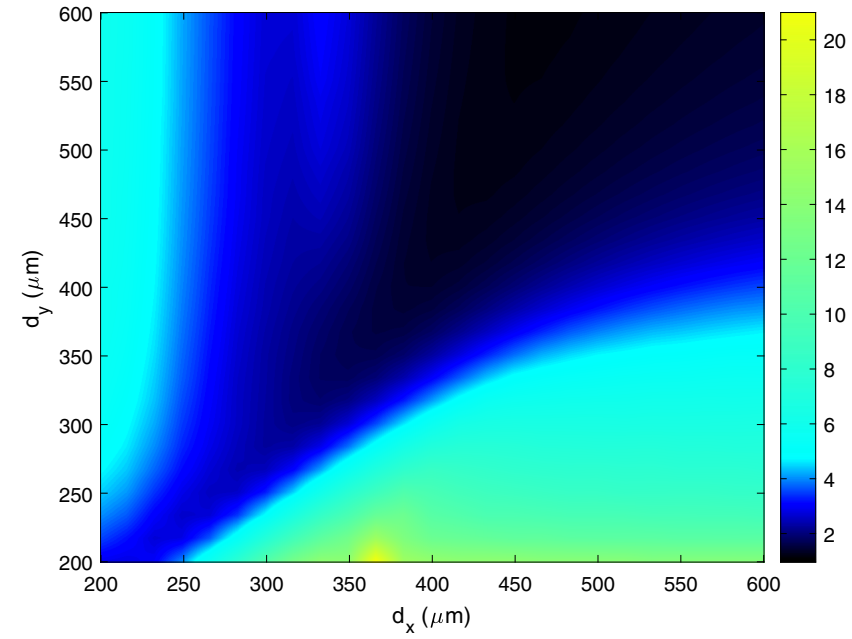

FIG. 3. The averaged mismatching factor $\bar{\Phi}$ in the lasing area of the bunch. Variables $d_{x}$ and $d_{y}$ are distances from the center of the bunch to jaws in the horizontal and vertical dechirpers, respectively. pulses. The time-dependent quadrupole wake components in the second dechirper may change the shape of the dipole kick produced in the first dechirper. An example is shown in Fig. 4. The bunch accumulates a dipole kick $\left\langle y_{V}^{\prime}(s)\right\rangle=$ $D_{V}(s)$ in the vertical dechirper. The time-dependent offset in $\langle y(s)\rangle$ can be modeled by $\langle y(s)\rangle=D_{V}(s) L / 2$, where $L$ is the length of the dechirper. Here, $\left\langle y^{\prime}(s)\right\rangle$ and $\langle y(s)\rangle$ are the averaged transverse offsets in the angle and position, respectively, of the slice at $s$.

After the first dechirper, there is a drift of length $L_{1}=$ $0.51 \mathrm{~m}$, a defocusing quadrupole magnet of focal length $f=7.15 \mathrm{~m}$, and another drift of length $L_{1}=0.51 \mathrm{~m}$. The dipole kick in $y$ at the entrance of the second dechirper can be described by

$$
\begin{aligned}
\langle y(s)\rangle & =D_{V}(s)\left[\frac{L}{2}+2 L_{1}+\frac{L_{1}\left(L / 2+L_{1}\right)}{f}\right], \\
\left\langle y^{\prime}(s)\right\rangle & =D_{V}(s)\left(1+\frac{L / 2+L_{1}}{f}\right) .
\end{aligned}
$$

Both $\langle y(s)\rangle$ and $\left\langle y^{\prime}(s)\right\rangle$ still have a quadraticlike form at the entrance of the second dechirper, as shown in Figs. 4(e) and 4(f). However, the downstream dechirper leads to a
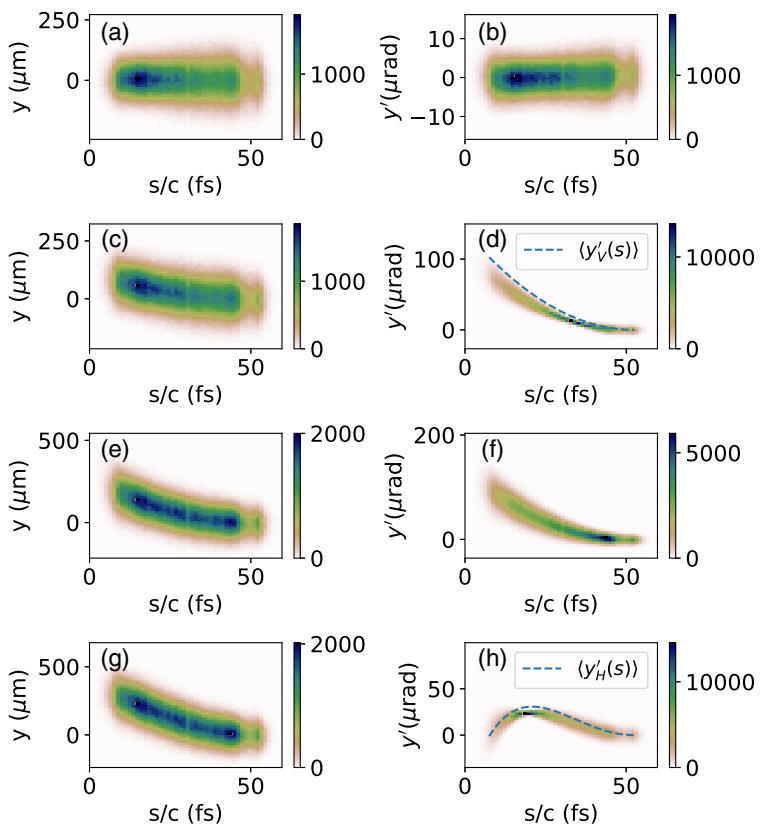

FIG. 4. With the dechirper setup $d_{x}=d_{y}=350 \mu \mathrm{m}, y-s$ and $y^{\prime}-s$ distributions of the bunch at (a),(b) the entrance of the first dechirper, (c),(d) the end of the first dechirper, (e),(f) the entrance of the second dechirper, and $(\mathrm{g}),(\mathrm{h})$ the end of the second dechirper. The center of the beam line is located at $x=y=0$. The head of the beam is on the right. Notice that the bunch longitudinal coordinate on the $x$ axis is shown in femtoseconds. Dashed lines in (d) and (h) show vertical angle offsets at the end of the vertical and the horizontal dechirper given by Eqs. (8) and (9), respectively. 
time-dependent kick in $y$. By assuming the bunch has a flattop longitudinal distribution of length $L_{b}$,

$$
\left\langle y_{V}^{\prime}(s)\right\rangle=D_{V}(s)=\frac{Z_{0} c}{4 \pi} \frac{e Q L}{E} \frac{\left(L_{b}-s\right)^{2}}{2 L_{b} d_{y}^{3}}
$$

The angle offset $\left\langle y^{\prime}(s)\right\rangle$ after the second dechirper calculated via Eqs. (A2) is given by

$\left\langle y_{H}^{\prime}(s)\right\rangle=D_{V}(s)\left(1+\frac{L / 2+L_{1}}{f}-\frac{Z_{0} c}{4 \pi} \frac{e Q L 5 L^{*}\left(L_{b}-s\right)^{2}}{8 L_{b} d_{x}^{4}}\right)$,

where the effective length $L^{*}$ is given by

$$
L^{*}=\left(L+2 L_{1}\right)\left(1+\frac{L+2 L_{1}}{4 f}\right)
$$

The derivation of Eq. (9) is given in Appendix B.

For LCLS parameters, the transverse kick $\left\langle y_{H}^{\prime}(s)\right\rangle=$ $D_{V}(s)\left[1.21-1.22\left(1-s / L_{b}\right)^{2}\right] \quad$ in our flattop-current approximation with $d_{x}=d_{y}=350 \mu \mathrm{m}$. We notice that the factor $\left[1.21-1.22\left(1-s / L_{b}\right)^{2}\right]$ decreases from 1.21 at the bunch head to -0.01 at the bunch tail. When $d_{x}$ is too small, the time-dependent focusing in the second dechirper could be too strong, and $\left\langle y^{\prime}(s)\right\rangle$ at the exit of the dechirper system may be no longer monotonic from the head to the tail of the bunch, as shown in Fig. 4(h). The nonmonotonic kick in one direction can make the fresh-slice setup more difficult, as it requires a careful setting of each transverse orbit in the undulator sections, and can be harmful when trying to achieve the shortest pulse duration. However, the effect may be useful if a setup requires more photons on the pulse produced on the bunch tail, by allowing a longer pulse.

Because of the CSR effect, the bunch may present a time-dependent tilt and angles in $x$ before the dechirper system. The CSR kick influences the shape of $\left\langle x^{\prime}(s)\right\rangle$ after the horizontal dechirper and can be harmful for the lasing suppression. As in the experiment, we rely more on the dipole kick in $y$ to perform the lasing suppression. Similarly to the experimental case, we first decrease $d_{y}$ until the vertical dipole kick is large enough for the lasing suppression. We then select the smallest $d_{x}$ to make sure that the mismatching effect can be canceled and $\left\langle y^{\prime}(s)\right\rangle$ is still monotonic after the horizontal dechirper. We choose our optimal dechirper setup to be $d_{x}=430 \mu \mathrm{m}$ and $d_{y}=380 \mu \mathrm{m}$. The angle offsets in the optimal setup are plotted in Fig. 5. Both $\left\langle x^{\prime}(s)\right\rangle$ and $\left\langle y^{\prime}(s)\right\rangle$ can maintain a quadratic shape pretty well after the transport from the dechirper system to the undulator entrance.
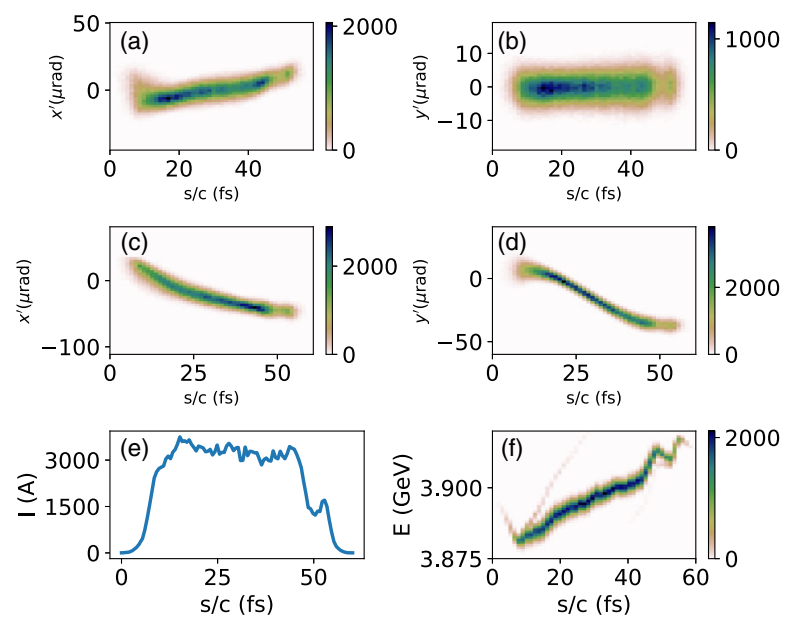

FIG. 5. (a) and (b) show the angle offsets $\left\langle x^{\prime}(s)\right\rangle$ and $\left\langle y^{\prime}(s)\right\rangle$ before the first dechirper. (c) and (d) show the angle offsets $x^{\prime}(s)$ and $y^{\prime}(s)$ and at the undulator entrance. The dechirper setup is $d_{x}=430 \mu \mathrm{m}$ and $d_{y}=380 \mu \mathrm{m}$. The tail of the beam is on the straight trajectory when the lasing slice control is done. (a) shows CSR kicks along the bunch in $x$. (e) shows the current, and (f) shows the $E-t$ distribution of the bunch at the undulator entrance. The chirp in the phase space is due to the timedependent energy loss in the dechirper system.

\section{B. Lasing slice control}

Two pairs of orbit correctors are used to control the electron bunch orbit in order to set the desired temporal slice to the straight on-axis trajectory suitable to lase in an undulator section.

Suppose that the averaged transverse position and angle of the slice at $s$ is $\vec{x}_{i}(s)=\left[\langle x(s)\rangle,\left\langle x^{\prime}(s)\right\rangle,\langle y(s)\rangle,\left\langle y^{\prime}(s)\right\rangle\right]^{T}$ before the first pair of kickers. The first pair of correctors gives the whole bunch a transverse kick $\Delta \vec{x}_{1}=$ $\left(0, \Delta x_{1}^{\prime}, 0, \Delta y_{1}^{\prime}\right)^{T}$ in the thin lens approximation. The beam transport between the two pairs of kickers can be described by the $4 \times 4$ matrix $M$. After the second pair of kickers, the slice average position and angles are described by

$$
\vec{x}_{f}(s)=M\left[\vec{x}_{i}(s)+\Delta \vec{x}_{1}\right]+\Delta \vec{x}_{2},
$$

where $\Delta \vec{x}_{2}=\left(0, \Delta x_{2}^{\prime}, 0, \Delta y_{2}^{\prime}\right)^{T}$ is the transverse kick from the second pair of kickers.

The corrector kicks required to set the slice on axis are calculated by solving $\vec{x}_{f}(s)=\overrightarrow{0}$ in the variables $\Delta x_{1}^{\prime}, \Delta y_{1}^{\prime}$, $\Delta x_{2}^{\prime}$, and $\Delta y_{2}^{\prime}$. The beam transport described by $M$ between two pairs of kickers must contain some drifts to make $\vec{x}_{f}(s)=\overrightarrow{0}$ solvable. In experiments, the orbit correction for the first undulator section is done by solving the setup of two pairs of kickers in the Linac to Undualtor (LTU) beam line. Orbit corrections for the second and third sections are done by setting up kickers before and after two chicanes in the undulator beam line. 
The slippage is significant for the short $\mathrm{x}$-ray pulses produced with the multistage scheme. For $670 \mathrm{eV}$ photons, the full slippage is $\sim 0.7 \mathrm{fs}$ in each undulator segment consisting of 110 undulator periods. From the second undulator section, the FEL process reaches saturation in one undulator segment, and, given the section length, the slippage is as large as $4 \mathrm{fs}$, comparable to or longer than the $\mathrm{x}$-ray pulse duration. Therefore, one cannot precisely infer which slice is on the straight trajectory in a certain undulator section from the time-resolved bunch energy losses measured downstream of the FEL process.

\section{XFEL pulse analysis}

We optimize the selection of on-axis slices in three undulator sections using the beam shown in Fig. 5 and the undulator strength setup shown in Fig. 6 based on the produced FEL spectra, favoring intense single spikes.

The optimal selection of on-axis slices is shown in Fig. 7. The optimal longitudinal distance between the first and the second on-axis slices is $10.5 \mathrm{fs}$, and the one between the second and the third on-axis slices is $9.1 \mathrm{fs}$, both longer than the chicane delay $4 \mathrm{fs}$, due to significant slippage lengths. The normalized XFEL power distribution along the bunch of the optimal configuration is shown in Fig. 8. The emission of undulator radiation, with the power $P(s)$ proportional to the longitudinal current density $I(s)$, is visible at the early stage of self-amplified spontaneous emission in the first section, as shown in the left side of Fig. 8. Then only the portion of the XFEL pulse overlapping with slices on almost straight trajectories can be further effectively amplified through the FEL exponential gain. Therefore, the temporal shape of the pulse quickly becomes cleaner when the bunch moves downstream.

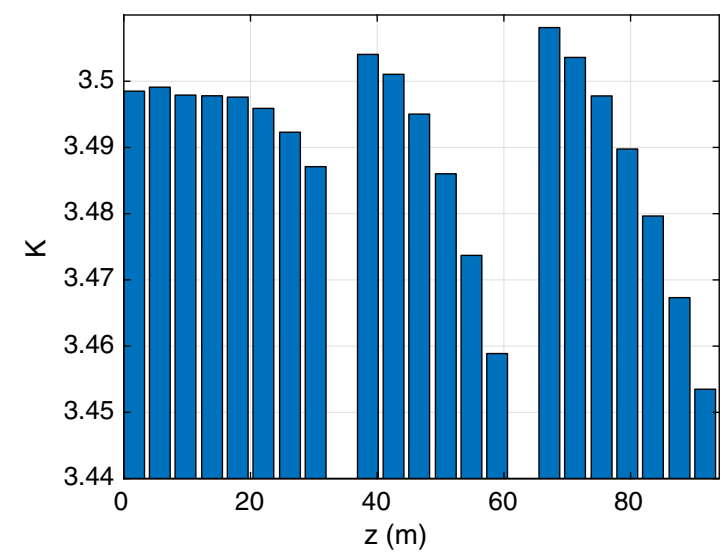

FIG. 6. The undulator strength parameter $K$ used in the freshslice multistage XFEL amplification experiment [11]. By gradually moving the on-axis slice to the head, the slice energy becomes higher, since the wake-induced energy loss becomes smaller from the bunch tail to the head. In order to meet the FEL resonance condition, the starting point of the undulator parameter becomes higher from the first to the third stage.
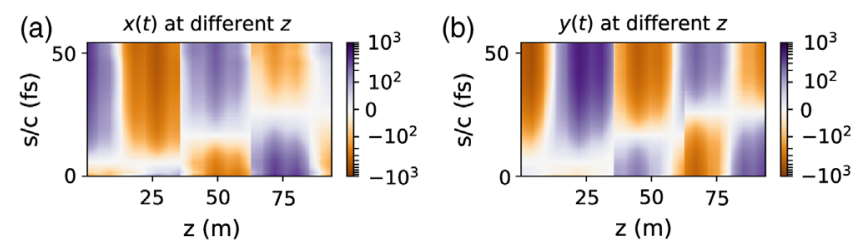

FIG. 7. At different positions $z$ in the undulator beam line, (a) the offset $x(s)$ in $\mathrm{X}$ and (b) the offset $y(s)$ in Y along the bunch in micrometers. The center of the beam line is located at $x=y=0$.

Because of the significant slippage for soft x-ray pulses, the location of the peak of the pulse moves around 3 fs to the bunch head compared to the location of the on-axis slice in the first undulator section. After the orbit correction and the 4 fs delay in the first chicane, the XFEL pulse moves toward the middle of the bunch and overlaps with fresh electrons that have not lased yet. The slippage of the pulse is around $4 \mathrm{fs}$ in the second stage. Then, after another orbit correction and another 4 fs delay in the second chicane, the XFEL pulse overlaps with and gets further amplified by onaxis fresh electrons in the middle of the bunch in the third stage.

It is worth noticing that the selection of the second and the third on-axis slices shown in Figs. 7 and 8 does not simply match the chicane delay shift. Our optimization shows that it is beneficial to select the on-axis slice slightly in front of the peak of the pulse at the start of both the second and the third stages. When the bunch moves downstream, the peak of the pulse moves toward the bunch head and sweeps through the on-axis slice due to the slippage, within the same undulator section. In this way, the peak of the pulse can be continuously amplified by the fresh on-axis slice. Because of the slippage, the pulse peak will quickly sweep away and have no more overlap with the on-axis slice, leading to little gain in the peak, if the on-axis slice is selected exactly at the peak of the pulse at the start of the undulator section.

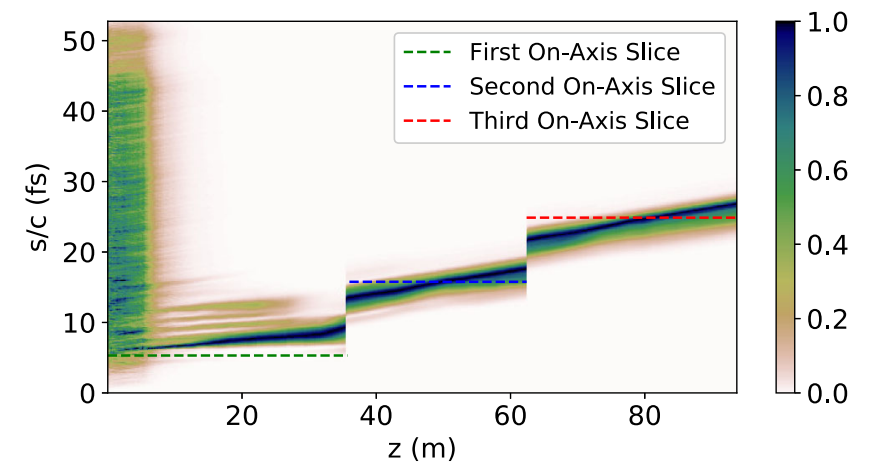

FIG. 8. The normalized XFEL power distribution $\tilde{P}(s)=$ $P(s) / \max _{s} P(s)$ along the bunch at different $z$ in the undulator beam line. The green, blue, and red dashed lines show the locations of on-axis slices in the first, second, and third undulator sections, respectively. 


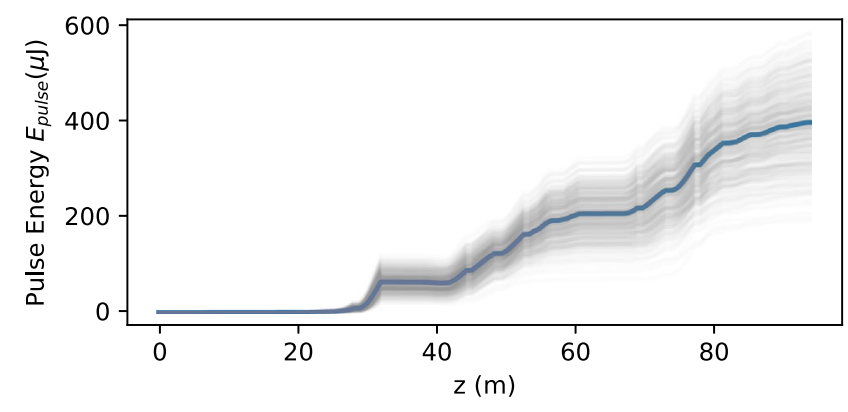

FIG. 9. Multiple-shot FEL pulse energy profile along the undulator beam line. The blue line shows the evolution of the averaged XFEL pulse energy $\bar{P}_{\text {pulse }}(z)$ in the undulator beam line.

We stop at the end of the seventh undulator module in the third stage. The lasing suppression is weak in the bunch head due to the flat slope of transverse offsets there. The FEL pulse will move further to the bunch head and make the whole bunch head radiate if we make use of all 15 undulator modules in the third stage. This can make the pulse become noisier and longer in the time domain. The single-spike performance in the frequency domain can be lost.

We perform 500 shots of GENESIS version 4 simulations with the optimal dechirper and lasing slice setup. The evolution of 500-shot FEL pulse energy along the undulator beam line is shown in Fig. 9. The pulse energy is $64 \pm 19,206 \pm 45$, and $397 \pm 78 \mu \mathrm{J}$ at the end of the first, the second, and the third stage, respectively.

The evolution of the XFEL pulse in both the frequency and the time domain for a single simulated shot is shown in Figs. 10(a)-10(f). At the end of the first stage, the pulse has a side peak in the frequency domain. By the next two stages of cascaded amplification, the pulse becomes single-spike Gaussian-like in both the frequency and the time domain. The generation of single-spike pulses in both the frequency and the time domain can benefit from strong undulator tapers in the second and the third stage, as shown in Fig. 6.

Only the portion of electrons overlapping with the peak of the XFEL pulse seed generated in the previous stage can quickly lose energy and continuously match the strong undulator taper. As a result, only the main peak of the XFEL pulse in the time domain can be effectively amplified, resulting in a more temporal single-spike pulse at the end. Figures 10(g)-10(i) show the evolution of the bunch phase space at the end of the first, the second, and the third stages. The bunch energy loss and energy spread induced by different stages of FEL radiation can be clearly verified.

The horizontal and vertical center-of-mass orbits of the bunch are shown in Figs. 11(a) and 11(b), respectively. Center-of-mass orbits are experimentally measured by cavity beam position monitors located between undulator segments. By selecting the first on-axis slice in the bunch tail, the oscillation amplitudes of horizontal and vertical center-ofmass orbits are both around $250 \mu \mathrm{m}$. These amplitudes get reduced to around $100 \mu \mathrm{m}$ in the second stage, since the second on-axis slice is selected to be closer to the bunch head than the first on-axis slice. Center-of-mass orbits become almost flat in the third stage, since the third on-axis slice is close to the center of the bunch. The machine model for the transfer line from the end of the linac to the undulator line (LTU) is not fully accurate, and, therefore, some discrepancy in the beam matching and phase advance between the dechirper and the undulator entrance is expected. In previous experiments requiring better accuracy of the beam transport model, such as the matching-based fresh slice [18], the beam
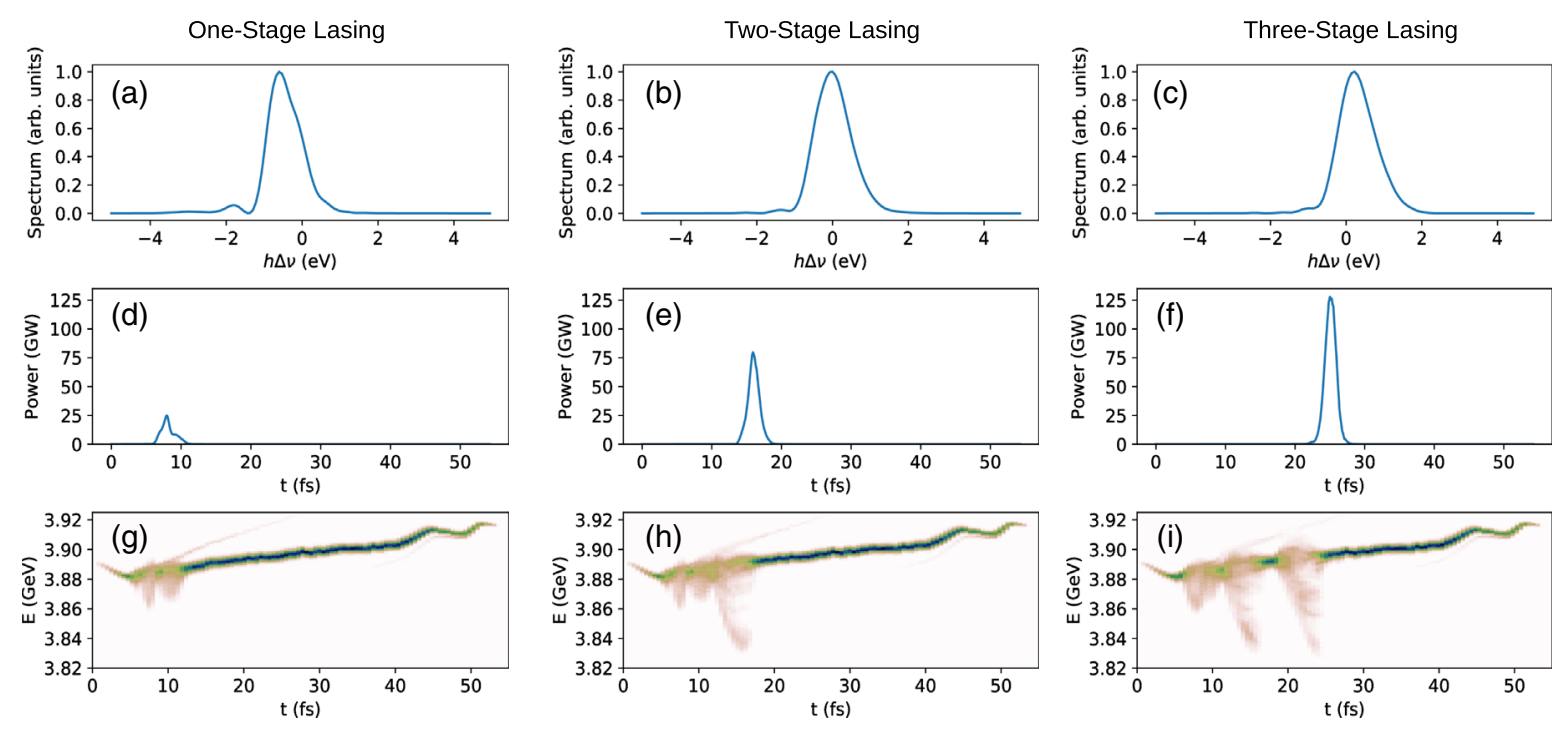

FIG. 10. (a)-(c) XFEL pulses in the frequency domain at the exit of the first, second, and third undulator sections. (d)-(f) XFEL pulses in the time domain at the exit of the first, second, and third undulator sections. (g)-(i) Phase space distributions of the electron bunch at the exit of the first, second, and third undulator sections. 

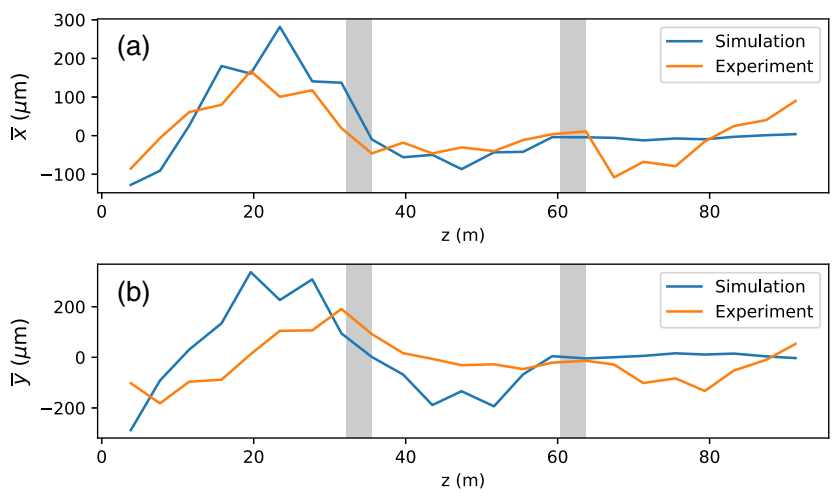

FIG. 11. Horizontal and vertical center-of-mass orbits of the bunch in the undulator line. Two gray blocks show the locations of two chicanes in the undulator beam line.

transport matrices were experimentally measured, and the measured model was used. However, for the multistage freshslice experiment, this procedure was not carried out.

Table II shows statistics of spike numbers in the frequency domain of 500 FEL simulations. The 24.0\% single-spike rate in the simulation is higher than $13.0 \%$ shown in Ref. [11]. In experiments, machine jitters in the LCLS can influence the temporal current of the bunch before the dechirper system. Transverse kicks produced in the dechirper system vary with different temporal current profiles $I(s)$, leading to inaccurate lasing slice controls by the designed setup of orbit correctors. As a result, experimental spectra can be less clean compared to ones in a simulation.

Figure 12 shows histograms with the distribution of relevant XFEL pulses properties at the end of the third stage. The pulse energy is $397 \pm 78 \mu \mathrm{J}$, and the spectral width is $1.17 \pm 0.46 \mathrm{eV}$. The FWHM temporal duration of $2.79 \pm 0.98 \mathrm{fs}$ has a large variability, because there are pulses presenting a very intense ultrashort feature of duration close to $1 \mathrm{fs}$, as well as pulses presenting a wider spike or a double-spike structure. For nonlinear pumpprobe experiments using XFEL pulses, the duration of the dominant temporal spike is typically a relevant quantity. The pulse duration $\Delta t_{74 \%}$ is defined as the shortest duration containing $74 \%$ of the pulse energy and was measured as $3.25 \pm 0.29$ fs. The difference between distributions shown in Figs. 10(c) and 10(d) is understood from the variability of the x-ray temporal profile. The peak power is

TABLE II. Statistics of XFEL pulses in the frequency domain of two dechirper configurations on 500 shots of simulations.

\begin{tabular}{lcc}
\hline \hline Spike count & Two-dechirper setup & One-dechirper setup \\
\hline 1 & $24.0 \%$ & $37.2 .0 \%$ \\
2 & $38.8 \%$ & $31.0 \%$ \\
3 & $22.6 \%$ & $14.6 .0 \%$ \\
$>3$ & $14.6 \%$ & $17.2 \%$ \\
\hline \hline
\end{tabular}
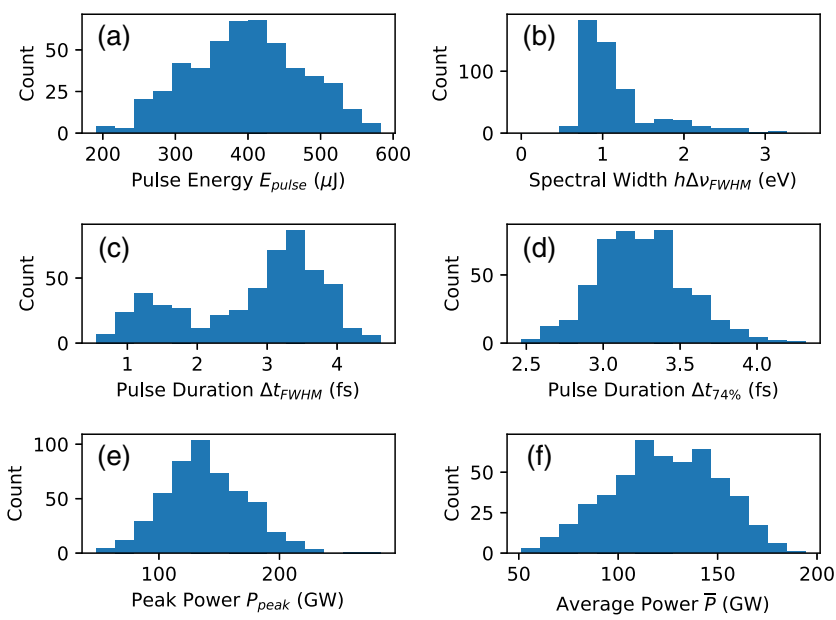

FIG. 12. Statistics of 500 shots of the simulation in Sec. III C. Histograms of (a) the pulse energies $E_{\text {pulse }}$, (b) the spectral widths $h \Delta \nu_{\text {FWHM }}$ defined as the full width at half maximum (FWHM) in the frequency domain, (c) the pulse durations $\Delta t_{\mathrm{FWHM}}$ defined as the FWHM in the time domain, (d) the pulse durations $\Delta t_{74 \%}$ defined as the shortest duration containing $74 \%$ of the pulse energy, (e) the peak powers $P_{\text {peak }}$, and (f) the average pulse power of each XFEL shot defined as $\bar{P}=E_{\text {pulse }} / \Delta t_{74 \%}$.
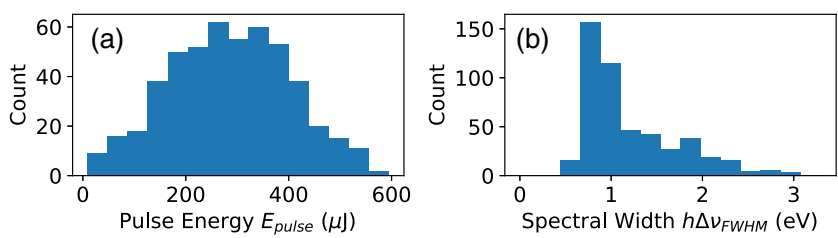

FIG. 13. Statistics of 500 shots of experiment data of Ref. [11]. (a) and (b) show histograms of the pulse energies $E_{\text {pulse }}$ and the FWHM spectral widths $h \Delta \nu_{\text {FWHM }}$, respectively.

$139 \pm 34 \mathrm{GW}$. The average power of each XFEL shot is $123 \pm 27 \mathrm{GW}$.

Histograms of the pulse energy and FWHM spectral width of three-stage FEL experiment data are shown in Fig. 13. The pulse energy is $287 \pm 117 \mu \mathrm{J}$ in the experiment. Compared to Fig. 12(a), the lower average value of the larger standard deviation of experimental pulse duration can be understood as a result of the machine jitters in the LCLS. The FWHM spectral width $1.25 \pm 0.61 \mathrm{eV}$ in experiment data is in good agreement with simulation results.

\section{A SIMPLER AND MORE ROBUST SCHEME}

The fresh-slice setup with two dechirpers may lead to a nonmonotonic dipole kick in one direction and be harmful for achieving the shortest pulse duration. The transverse slice focusing interplay between the two devices and the necessity of installing two modules make the possibility of using a single device appealing. As a result, we discuss an alternative scheme for the fresh-slice setup using a single 
vertical dechirper identical to the existing one. We consider, however, a different transverse matching setup.

With only one dechirper, the time-dependent mismatching produced in the dechirper can no longer be cancelled in the downstream beam line. Hence, in order to make the mismatching effect less harmful, we need to make the transverse size of the bunch to be small at the entrance of the dechirper system. The correlation between the transverse position and angle, described by the Twiss parameter $\alpha$, should also be small to reduce the mismatching effect. In our simulation, we track the bunch in ELEGANT using LCLS parameters till the entrance of the dechirper system in the experiment. We then transversely scale the beam to $\alpha_{x}=\alpha_{y}=0$ and $\beta_{x}=\beta_{y}=4 \mathrm{~m}$. Downstream of the dechirper, we consider the same matching section as in the LCLS and a lasing slice control beam line. When dechirper jaws are fully retracted, focusing strengths of four matching quadrupole magnets in LTU are tuned to match the scaled beam to the designed optics, listed in Table I, at the undulator entrance. The electron bunch is further tracked till the undulator entrance in ELEGANT using our designed beam line, with the vertical dechirper jaw in. The lasing slice control is optimized towards producing high-power single spikes.

With such a small bunch, a setting of $d_{y}=310 \mu \mathrm{m}$ in the vertical dechirper presents still an averaged mismatching factor $\bar{\Phi}=1.83$, which can allow a good soft $\mathrm{x}$-ray FEL performance. The transverse offset of the beam at the undulator entrance is shown in Fig. 14. By comparing Figs. 4(h) and 14(b), we can see that the dipole kick at the bunch tail with the single-dechirper setup is quadratic and monotonic and has a larger slope. A monotonic transverse kick with a larger slope can lead to a better lasing suppression in the surrounding longitudinal area of the on-axis slice, which is expected to generate $\mathrm{x}$-ray pulses with a shorter duration.

We optimize undulator strengths and locations of on-axis slices in all three undulator sections. The optimal undulator strengths are shown in Fig. 15. We start to taper the $K$ in a quadratic form from the fifth undulator module in the first stage. Quadratic tapers start from the first undulator module in both the second and the third stage. The longitudinal distance between the first and the second on-axis slices is
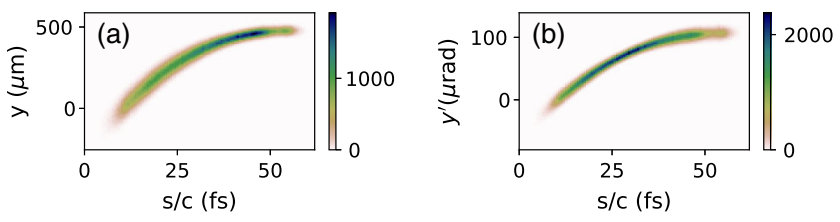

FIG. 14. With the single-dechirper setup $d_{y}=310 \mu \mathrm{m}$, (a) the $y-t$ distribution and (b) the $y^{\prime}-t$ distribution of the bunch at the undulator entrance. Notice that the tail of the beam is on the straight trajectory, since the lasing slice control for the first undulator section has been done.

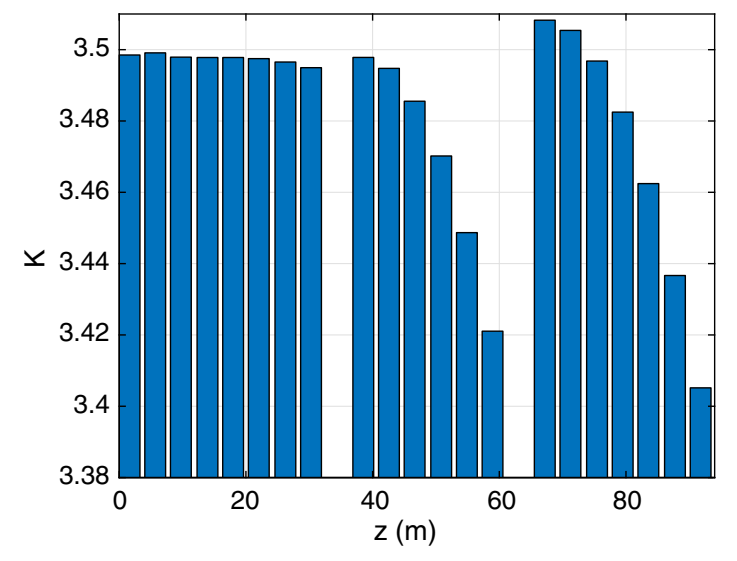

FIG. 15. The undulator strength parameter $K$ used in the singledechirper setup. The undulator strengths of first four undulator segments in the first undulator section are the same as the ones used in Ref. [11].

$10.5 \mathrm{fs}$, and the one between the second and the third on-axis slices is 6.5 fs.

The quadratic time-dependent transverse offset leads to a better lasing suppression in a bunch. As a result, the averaged temporal pulse duration of the single-dechirper setup is shorter than the one of the double-dechirper setup at the same $z$ in the undulator beam line, and the undulator taper stronger than the one of the double-dechirper setup to maintain the shorter temporal duration in the singledechirper setup, which can be verified by comparing Figs. 6 and 15.
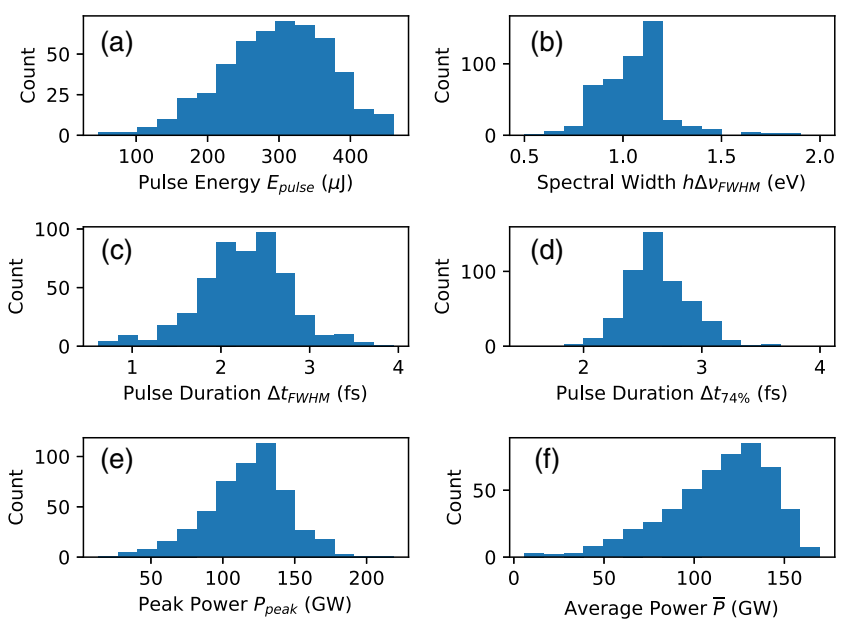

FIG. 16. Statistics of 500 shots simulation in the singledechirper setup. Histograms of (a) the pulse energies $E_{\text {pulse }}$, (b) the spectral widths $h \Delta \nu_{\text {FWHM }}$ defined as the FWHM in the frequency domain, (c) the pulse durations $\Delta t_{\mathrm{FWHM}}$ defined as the FWHM in the time domain, (d) the pulse durations $\Delta t_{74 \%}$ defined as the shortest duration containing $74 \%$ of the pulse energy, (e) the peak powers $P_{\text {peak }}$, and (f) the average pulse power of each XFEL shot defined as $\bar{P}=E_{\text {pulse }} / \Delta t_{74 \%}$. 

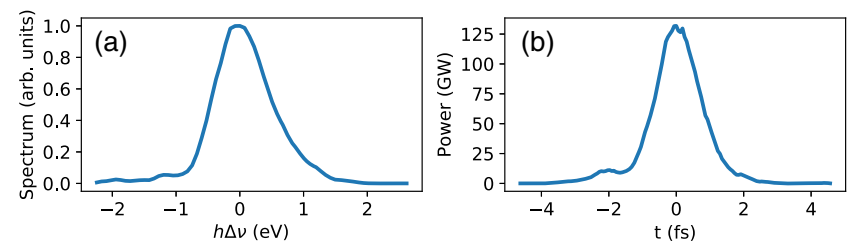

FIG. 17. (a) and (b) show one shot of the simulation with the single-dechirper setup in the spectral and the time domain, respectively.

We run 500 shots of GENESIS version 4 simulations with the optimal setup. Distributions of relevant XFEL pulses properties at the end of the third stage in the singledechirper setup are shown in Fig. 16. The pulse energy is $297 \pm 75 \mu \mathrm{J}$, and the spectral width is $1.10 \pm 0.25 \mathrm{eV}$. The FWHM temporal duration is $2.26 \pm 0.50 \mathrm{fs}$, and pulse duration $\Delta t_{74 \%}$ defined as the shortest duration containing $74 \%$ of the pulse energy is $2.67 \pm 0.41 \mathrm{fs}$. We notice that there is no longer a significant side peak located at around 1.2 fs in Fig. 16(c), in contrast to Fig. 12(c). Both histograms in Figs. 16(c) and 16(d) have only one significant peak. This shows that the generation of powerful short single-spike pulses can be achieved with the singledechirper setup and yields also more stable longitudinal $\mathrm{x}$-ray profiles. The latter is an important feature to make the experimental data interpretation easier. The peak power is $117 \pm 29 \mathrm{GW}$. The average power of each XFEL shot is $113 \pm 29 \mathrm{GW}$. One single-spike shot at the end of the third undulator section is shown in Fig. 17. Statistics of numbers of spikes in the frequency domain are shown in Table II.

\section{CONCLUSION}

We have developed a start-to-end simulation to analyze and optimize the fresh-slice multistage FELs. By optimizing the setup of the double-dechirper system, the timedependent mismatching along the beam can be canceled pretty well, and the transverse dipole kick can still maintain a monotonic shape to enable the fresh-slice operation for short pulse generation. Orbit correctors can be optimized to bring a certain slice in the bunch back to the straight trajectory, making the slice effectively amplify the FEL pulse in the downstream undulator beam line. With LCLS parameters, we perform simulations of the three-stage $670 \mathrm{eV}$ x-ray FEL amplification, the results of which are in good agreement with data in Ref. [11]. We show that the peak power of FEL pulses can exceed $200 \mathrm{GW}$ for the brightest shots and the averaged pulse duration is around 3.3 fs.

Our study reveals potential difficulties in operating a long dechirper system consisting of many dechirper modules. The wake-induced quadrupole effect can make the transverse kick nonmonotonic, resulting in difficult lasing slice controls. As a comparison, we show that the multistage fresh-slice setup can be further realized with an alternative scheme using only one dechirper and a strongly focused beam at the dechirper entrance. With the larger dipole kick, especially at the bunch tail, in the single-dechirper setup, the pulse duration can be further shortened to around $2.3 \mathrm{fs}$ with similar pulse power. The single-dechirper setup may lead to a more robust scheme to generate ultrashort $\mathrm{x}$-ray pulses and to improve the single-spike FEL performance.

\section{ACKNOWLEDGMENTS}

We thank Gennady V. Stupakov, Karl L. Bane, Gabriel Marcus, James P. MacArthur, Siqi Li, and Jingyi Tang for useful discussions. This work was supported by the U.S. Department of Energy, Office of Science, Laboratory Directed Research and Development (LDRD) program at SLAC National Accelerator Laboratory, under Contract No. DE-AC02-76SF00515. Z. G. acknowledges support from the Robert H. Siemann Graduate Fellowship in Physics.

\section{APPENDIX A: THE TRANSVERSE MOMENTUM KICK IN SINGLE-JAW DECHIRPERS}

Here, we take the wake-induced transverse kick in the horizontal dechirper as an example. The horizontal dipole kick of the test particle at $(x, y, s)$ in the horizontal dechirper is given by the convolution between the point wake in Eqs. (1) and the particle density distribution:

$$
\begin{aligned}
\Delta x_{H}^{\prime}(x, y, s)= & \frac{Z_{0} c}{4 \pi} \frac{e Q L}{E} \int_{-\infty}^{+\infty} d \tilde{x} \int_{-\infty}^{+\infty} d \tilde{y} \int_{0}^{+\infty} d \zeta \\
& \times w_{H, x}(x, y, \tilde{x}, \tilde{y}, \zeta) \rho(\tilde{x}, \tilde{y}, s+\zeta)
\end{aligned}
$$

where $Z_{0}=377 \Omega$ is the vacuum impedance, $Q$ is the total charge of the bunch, $L$ is the length of the corrugated plate, and $\rho(x, y, s)$ is the 3D density distribution normalized to 1 . The kick $\Delta y_{H}^{\prime}(x, y, s)$ in $y$ is similar by changing $w_{H, x}(x, y, \tilde{x}, \tilde{y}, \zeta)$ to $w_{H, y}(x, y, \tilde{x}, \tilde{y}, \zeta)$ in Eq. (A1).

The transverse kicks of the test particle in the horizontal dechirper can be simplified to

$$
\begin{aligned}
& \Delta x_{H}^{\prime}(x, y, s)=D_{H}(s)+x \cdot f_{H, q}^{-1}(s)-Q_{H, x}(s), \\
& \Delta y_{H}^{\prime}(x, y, s)=-y \cdot f_{H, q}^{-1}(s)+Q_{H, y}(s) .
\end{aligned}
$$

Here, the time-dependent dipole kick $D_{H}(s)$, the quadrupole focusing length $f_{H, q}(s)$, and transverse kicks $Q_{H, x}(s)$ and $Q_{H, y}(s)$ coming from quadrupole wake effects in the horizontal dechirper are given by following convolutions: 


$$
\begin{aligned}
D_{H}(s) & =\frac{Z_{0} c}{4 \pi} \frac{e Q L}{E} \int_{0}^{+\infty} d \zeta w_{H, d}(\zeta) \lambda(s+\zeta), \\
f_{H, q}^{-1}(s) & =\frac{Z_{0} c}{4 \pi} \frac{e Q L}{E} \int_{0}^{+\infty} d \zeta w_{H, q}(\zeta) \lambda(s+\zeta), \\
Q_{H, x}(s) & =\frac{Z_{0} c}{4 \pi} \frac{e Q L}{E} \int_{0}^{+\infty} d \zeta\langle x(s+\zeta)\rangle w_{H, q}(\zeta) \lambda(s+\zeta), \\
Q_{H, y}(s) & =\frac{Z_{0} c}{4 \pi} \frac{e Q L}{E} \int_{0}^{+\infty} d \zeta\langle y(s+\zeta)\rangle w_{H, q}(\zeta) \lambda(s+\zeta),
\end{aligned}
$$

where $E$ is the central energy of the bunch and $\lambda(s)=$ $\int d x \int d y \rho(x, y, s)$ is the longitudinal density distribution of the beam. The center-of-mass transverse position $[\langle x(s)\rangle,\langle y(s)\rangle]$ of elections in slice at $s$ is given by

$$
\begin{aligned}
\langle x(s)\rangle & =\frac{\int_{-\infty}^{+\infty} d \tilde{x} \int_{-\infty}^{+\infty} d \tilde{y} \rho(\tilde{x}, \tilde{y}, s+\zeta) \tilde{x}}{\lambda(s)}, \\
\langle y(s)\rangle & =\frac{\int_{-\infty}^{+\infty} d \tilde{x} \int_{-\infty}^{+\infty} d \tilde{y} \rho(\tilde{x}, \tilde{y}, s+\zeta) \tilde{y}}{\lambda(s)} .
\end{aligned}
$$

We can also define the center-of-mass angle $\left[\left\langle x^{\prime}(s)\right\rangle\right.$, $\left.\left\langle y^{\prime}(s)\right\rangle\right]$ of the slice at $s$ in the same way. The wake formulation for the vertical dechirper is the same as for the horizontal one, but switching $x$ and $y$ in the notation:

$$
\begin{aligned}
& \Delta x_{V}^{\prime}(x, y, s)=-x \cdot f_{V, q}^{-1}(s)+Q_{V, x}(s), \\
& \Delta y_{V}^{\prime}(x, y, s)=D_{V}(s)+y \cdot f_{V, q}^{-1}(s)-Q_{V, y}(s) .
\end{aligned}
$$

Parameters $D_{V}(s), f_{V, q}(s), Q_{V, x}(s)$, and $Q_{V, y}(s)$ are defined in the same way as Eqs. (A3) by using singleparticle wake functions $w_{V, d}(\zeta)$ and $w_{V, q}(\zeta)$ of the vertical dechirper.

By neglecting the CSR effect before the bunch enters the first (vertical) dechirper, say, $\langle x(s)\rangle=\langle y(s)\rangle=0$ for all $s$, Eqs. (A5) can be simplified to

$$
\begin{aligned}
& \Delta x_{V}^{\prime}(x, y, s)=-x \cdot f_{V, q}^{-1}(s), \\
& \Delta y_{V}^{\prime}(x, y, s)=D_{V}(s)+y \cdot f_{V, q}^{-1}(s) .
\end{aligned}
$$

The time-dependent quadrupole effect can be described by the matrix

$$
M_{V, q}(s)=\left(\begin{array}{cccc}
1 & 0 & 0 & 0 \\
-f_{V, q}^{-1}(s) & 1 & 0 & 0 \\
0 & 0 & 1 & 0 \\
0 & 0 & f_{V, q}^{-1}(s) & 1
\end{array}\right) \text {. }
$$

Besides the vertical dipole kick, the vertical dechirper leads to a time-dependent focusing effect in $x$ and a timedependent defocusing effect in $y$. Given the transverse trace space $\vec{x}_{i}=\left(x, x^{\prime}, y, y^{\prime}\right)^{T}$ of a particle at $(x, y, s)$ before the vertical dechirper of length $L$, the transverse trace space $\vec{x}_{f}$ of this electron after the vertical dechirper can be modeled by

$$
\vec{x}_{f}=M_{L / 2}\left[M_{V, q}(s) M_{L / 2} \vec{x}_{i}+\Delta \vec{x}_{V}\right],
$$

where $M_{L / 2}$ is the matrix of a drift of length $L / 2$ and $\Delta \vec{x}_{V}=\left[0,0,0, D_{V}(s)\right]^{T}$ is the vertical dipole kick.

The wakefield-induced transverse kick in the second dechirper is more complicated. The bunch enters the second dechirper with all slices not on the axis, leading to nonzero terms $Q_{H, x}(s)$ and $Q_{H, y}(s)$ in Eqs. (A2). This will be calculated in Appendix B on a flattop current model.

\section{APPENDIX B: THE NONQUADRATIC TRANSVERSE KICK IN THE DOUBLE- DECHIRPER CONFIGURATION}

Here, we model our bunch shown in Fig. 5 as a uniform flattop distribution:

$$
\lambda(s)= \begin{cases}1 / L_{b}, & \text { if } L_{b} \geq s \geq 0, \\ 0, & \text { otherwise, }\end{cases}
$$

where $L_{b} \sim 12 \mu \mathrm{m}$ is the bunch length. For the common dechirper setup $d_{x} \simeq d_{y} \simeq 400 \mu \mathrm{m}$, characteristic lengths $s_{0 x}$ and $s_{0 y}$ of the horizontal and the vertical dechirper are around $100 \mu \mathrm{m}$, which is one order of magnitude longer than the bunch length $L_{b}$. In this case, wake functions of two dechirpers described by Eqs. (2) and (3) can be greatly simplified to

$$
\begin{aligned}
w_{H, d}(\zeta) & \simeq \frac{2 s_{0 x}}{d_{x}^{3}}\left[1-\left(1+\sqrt{\frac{\zeta}{s_{0 x}}}\right)\left(1-\sqrt{\frac{\zeta}{s_{0 x}}}+\frac{\zeta}{2 s_{0 x}}\right)\right] \\
& \simeq \frac{\zeta}{d_{x}^{3}}, \\
w_{H, q}(\zeta) & \simeq \frac{3 s_{0 x}}{d_{x}^{4}}\left[1-\left(1+\sqrt{\frac{\zeta}{s_{0 x}}}\right)\left(1-\sqrt{\frac{\zeta}{s_{0 x}}}+\frac{\zeta}{2 s_{0 x}}\right)\right] \\
& \simeq \frac{3 \zeta}{2 d_{x}^{4}} .
\end{aligned}
$$

Similarly, $w_{V, d}(\zeta)=\zeta / d_{y}^{3}$ and $w_{V, q}(\zeta)=3 \zeta / 2 d_{y}^{4}$. With the above approximations, the vertical dipole kick $D_{V}(s)$ in the first dechirper can be given in an analytical solution:

$$
\begin{aligned}
D_{V}(s) & =\frac{Z_{0} c}{4 \pi} \frac{e Q L}{E} \int_{0}^{+\infty} d \zeta w_{V, d}(\zeta) \lambda(s+\zeta) \\
& =\frac{Z_{0} c}{4 \pi} \frac{e Q L}{E} \frac{\left(L_{b}-s\right)^{2}}{2 L_{b} d_{y}^{3}},
\end{aligned}
$$

which is in a quadratic form. 
We model the wake effect of the horizontal dechirper of length $L$ on the bunch in the following way: The bunch first goes through a free space drift of length $L / 2$, then gets transverse kicks described by Eqs. (A2) and (A3), and then goes through another free space drift of length $L / 2$. By propagating the transverse dipole kick in Eqs. (7) of length $L / 2$, the transverse offsets in the space and the angle are, respectively,

$$
\begin{aligned}
& \langle y(s)\rangle=D_{V}(s)\left(L+2 L_{1}\right)\left(1+\frac{L+2 L_{1}}{4 f}\right), \\
& \left\langle y^{\prime}(s)\right\rangle=D_{V}(s)\left(1+\frac{L / 2+L_{1}}{f}\right) .
\end{aligned}
$$

We focus on the averaged vertical kick $\left\langle\Delta y_{H}^{\prime}(s)\right\rangle$ of a slice at $s$ in the horizontal dechirper:

$$
\begin{aligned}
\left\langle\Delta y_{H}^{\prime}(s)\right\rangle= & -\frac{Z_{0} c}{4 \pi} \frac{e Q L}{E} \int_{0}^{+\infty} d \zeta[\langle y(s)\rangle \\
& -\langle y(s+\zeta)\rangle] w_{H, q}(\zeta) \lambda(s+\zeta) \\
= & -\left(\frac{Z_{0} c}{4 \pi} \frac{e Q L}{E}\right)^{2} \frac{5 L^{*}\left(L_{b}-s\right)^{4}}{8 L_{b}^{2} d_{x}^{4} d_{y}^{3}},
\end{aligned}
$$

where the effective length $L^{*}$ is given by

$$
L^{*}=\left(L+2 L_{1}\right)\left(1+\frac{L+2 L_{1}}{4 f}\right)
$$

The minus sign in the last line of Eqs. (B4) shows that the horizontal dechirper leads to a time-dependent kick in $y$. The angle offset in $y$ along the bunch at the end of the horizontal dechirper is

$$
\begin{aligned}
\left\langle y_{H}^{\prime}(s)\right\rangle= & D_{V}(s)\left(1+\frac{L / 2+L_{1}}{f}\right)+\left\langle\Delta y_{H}^{\prime}(s)\right\rangle \\
= & D_{V}(s)\left(1+\frac{L / 2+L_{1}}{f}\right. \\
& \left.-\frac{Z_{0} c}{4 \pi} \frac{e Q L}{E} \frac{5 L^{*}\left(L_{b}-s\right)^{2}}{8 L_{b} d_{x}^{4}}\right) .
\end{aligned}
$$

[1] P. Emma, R. Akre, J. Arthur, R. Bionta, C. Bostedt, J. Bozek, A. Brachmann, P. Bucksbaum, R. Coffee, F.-J. Decker et al., First lasing and operation of an ångstromwavelength free-electron laser, Nat. Photonics 4, 641 (2010).

[2] T. Ishikawa, H. Aoyagi, T. Asaka, Y. Asano, N. Azumi, T. Bizen, H. Ego, K. Fukami, T. Fukui, Y. Furukawa et al., A compact $\mathrm{X}$-ray free-electron laser emitting in the subångström region, Nat. Photonics 6, 540 (2012).
[3] E. Allaria, R. Appio, L. Badano, W. Barletta, S. Bassanese, S. Biedron, A. Borga, E. Busetto, D. Castronovo, P. Cinquegrana et al., Highly coherent and stable pulses from the fermi seeded free-electron laser in the extreme ultraviolet, Nat. Photonics 6, 699 (2012).

[4] M. Altarelli, R. Brinkmann, and M. Chergui, The European $\mathrm{X}$-ray free-electron laser. Technical design report, technical report, DEsY XFEL Project Group, 2007.

[5] W. A. Ackermann, G. Asova, V. Ayvazyan, A. Azima, N. Baboi, J. Bähr, V. Balandin, B. Beutner, A. Brandt, A. Bolzmann et al., Operation of a free-electron laser from the extreme ultraviolet to the water window, Nat. Photonics $\mathbf{1}$, 336 (2007).

[6] H.-S. Kang, C.-K. Min, H. Heo, C. Kim, H. Yang, G. Kim, I. Nam, S. Y. Baek, H.-J. Choi, G. Mun et al., Hard X-ray free-electron laser with femtosecond-scale timing jitter, Nat. Photonics 11, 708 (2017).

[7] C. Bostedt, S. Boutet, D. M. Fritz, Z. Huang, H. J. Lee, H. T. Lemke, A. Robert, W. F. Schlotter, J. J. Turner, and G. J. Williams, Linac coherent light source: The first five years, Rev. Mod. Phys. 88, 015007 (2016).

[8] A. A. Lutman, T. J. Maxwell, J. P. MacArthur, M. W. Guetg, N. Berrah, R. N. Coffee, Y. Ding, Z. Huang, A. Marinelli, S. Moeller et al., Fresh-slice multicolour X-ray free-electron lasers, Nat. Photonics 10, 745 (2016).

[9] A. A. Lutman, R. Coffee, Y. Ding, Z. Huang, J. Krzywinski, T. Maxwell, M. Messerschmidt, and H.-D. Nuhn, Experimental Demonstration of Femtosecond TwoColor X-Ray Free-Electron Lasers, Phys. Rev. Lett. 110, 134801 (2013).

[10] C. Emma, A. Lutman, M. Guetg, J. Krzywinski, A. Marinelli, J. Wu, and C. Pellegrini, Experimental demonstration of fresh bunch self-seeding in an X-ray free electron laser, Appl. Phys. Lett. 110, 154101 (2017).

[11] A. A. Lutman, M. W. Guetg, T. J. Maxwell, J. P. MacArthur, Y. Ding, C. Emma, J. Krzywinski, A. Marinelli, and Z. Huang, High-Power Femtosecond Soft X Rays from Fresh-Slice Multistage Free-Electron Lasers, Phys. Rev. Lett. 120, 264801 (2018).

[12] E. Prat, S. Bettoni, and S. Reiche, Enhanced X-ray free-electron-laser performance from tilted electron beams, Nucl. Instrum. Methods Phys. Res., Sect. A 865, 1 (2017).

[13] W. Qin, Y. Ding, A. A. Lutman, and Y.-C. Chao, Matchingbased fresh-slice method for generating two-color X-ray free-electron lasers, Phys. Rev. Accel. Beams 20, 090701 (2017).

[14] Z. Zhang, J. Duris, J. P. MacArthur, Z. Huang, and A. Marinelli, Double chirp-taper x-ray free-electron laser for attosecond pump-probe experiments, Phys. Rev. Accel. Beams 22, 050701 (2019).

[15] K. Bane and G. Stupakov, Corrugated pipe as a beam dechirper, Nucl. Instrum. Methods Phys. Res., Sect. A 690, 106 (2012).

[16] M. Guetg et al., Commissioning of the RadiaBeam/SLAC Dechirper, in Proceedings of the 7th International Particle Accelerator Conference (IPAC 2016), Busan, Korea, 2016 (JACoW, Geneva, Switzerland, 2016), MOPOW044, https://doi.org/10.18429/JACoW-IPAC2016-MOPOW044. 
[17] M. W. Guetg, A. A. Lutman, Y. Ding, T. J. Maxwell, and Z. Huang, Dispersion-Based Fresh-Slice Scheme for Free-Electron Lasers, Phys. Rev. Lett. 120, 264802 (2018).

[18] Y.-C. Chao, W. Qin, Y. Ding, A. A. Lutman, and T. Maxwell, Control of the Lasing Slice by Transverse Mismatch in an X-Ray Free-Electron Laser, Phys. Rev. Lett. 121, 064802 (2018).

[19] N. Hartmann, G. Hartmann, R. Heider, M. Wagner, M. Ilchen, J. Buck, A. Lindahl, C. Benko, J. Grünert, J. Krzywinski et al., Attosecond time-energy structure of x-ray free-electron laser pulses, Nat. Photonics 12, 215 (2018).

[20] C. Behrens, F.-J. Decker, Y. Ding, V. Dolgashev, J. Frisch, Z. Huang, P. Krejcik, H. Loos, A. Lutman, T. Maxwell et al., Few-femtosecond time-resolved measurements of X-ray free-electron lasers, Nat. Commun. 5, 3762 (2014).

[21] K. Bane, G. Stupakov, and I. Zagorodnov, Wakefields of a beam near a single plate in a flat dechirper, arXiv:1611 .09460 .
[22] K. Bane and G. Stupakov, Dechirper wakefields for short bunches, Nucl. Instrum. Methods Phys. Res., Sect. A 820, 156 (2016).

[23] P. Craievich and A. A. Lutman, Effects of the quadrupole wakefields in a passive streaker, Nucl. Instrum. Methods Phys. Res., Sect. A 865, 55 (2017).

[24] M. Borland, Elegant: A flexible SDDS-compliant code for accelerator simulation, technical report, Argonne National Lab, 2000.

[25] S. Reiche, Genesis 1.3: A fully 3d time-dependent FEL simulation code, Nucl. Instrum. Methods Phys. Res., Sect. A 429, 243 (1999).

[26] S. Reiche, Update on the fel code genesis 1.3, in Proceedings of the 2014 FEL Conference (JACoW, Geneva, Switzerland, 2014).

[27] J. Qiang, R. D. Ryne, S. Habib, and V. Decyk, An objectoriented parallel particle-in-cell code for beam dynamics simulation in linear accelerators, J. Comput. Phys. 163, 434 (2000).

[28] Y.-C. Chao, Distributed matching scheme and a flexible deterministic matching algorithm for arbitrary systems, arXiv:1704.08813 (2017). 